\title{
The Invariant Measure for the Two-Dimensional Parry-Daniels Map
}

\author{
By \\ Fritz Schweiger \\ (Vorgelegt in der Sitzung der math.-nat. Klasse am 15. November 2007 \\ durch das k. M. I. Fritz Schweiger)
}

\begin{abstract}
The Parry-Daniels map $T$ has an exceptional set $\Gamma$ which can be seen as a strange attractor for $T$. The density of the invariant measure is given. Some remarks on the exceptional set for the mixture of the Selmer algorithm and the fully subtractive algorithm are added.
\end{abstract}

Mathematics Subject Classification (2000): 11K55, 28D99.

Key words: Ergodic theory, invariant measures.

Let $x=\left(x_{0}, x_{1}, x_{2}\right) \in\left(\mathbb{R}^{+}\right)^{3}$ and let $\pi$ be a permutation of the indices such that $x_{\pi 0} \leq x_{\pi 1} \leq x_{\pi 2}$. The Poincaré map $P$ is defined as

$$
P\left(x_{0}, x_{1}, x_{2}\right)=\left(x_{\pi 0}, x_{\pi 1}-x_{\pi 0}, x_{\pi 2}-x_{\pi 1}\right) .
$$

We introduce

$$
\Sigma^{2}=\left\{x \in\left(\mathbb{R}^{+}\right)^{3}: x_{0}+x_{1}+x_{2}=1\right\} .
$$

Then the Parry-Daniels map $T: \Sigma^{2} \rightarrow \Sigma^{2}$ is defined as

$$
\begin{aligned}
T\left(x_{0}, x_{1}, x_{2}\right) & =\left(\frac{x_{\pi 0}}{x_{\pi 2}}, \frac{x_{\pi 1}-x_{\pi 0}}{x_{\pi 2}}, \frac{x_{\pi 2}-x_{\pi 1}}{x_{\pi 2}}\right), \\
\pi & \in\{\varepsilon,(01),(02),(12),(012),(021)\} .
\end{aligned}
$$


We introduce the notation

$$
x^{(k)}=\left(x_{0}^{(k)}, x_{1}^{(k)}, x_{2}^{(k)}\right):=P^{k} x .
$$

We define

$$
\sigma(x):=\sum_{k \geq 0} \max \left(x_{0}^{(k)}, x_{1}^{(k)}\right) .
$$

The following result could be proved (SCHWEIGER [2], NOGUEIRA [1]). Let

$$
\Gamma:=\bigcap_{s=0}^{\infty} \bigcup_{\pi_{1}, \ldots, \pi_{s} \in\{\varepsilon,(01)\}} B\left(\pi_{1}, \ldots, \pi_{s}\right),
$$

then $\Gamma=\left\{x \in \Sigma^{2}: \sigma(x) \leq x_{2}\right\}$ and $\lambda(\Gamma)>0$. Since $T$ is ergodic with respect to Lebesgue measure, we obtain

$$
\Sigma^{2}=\bigcup_{j=0}^{\infty} T^{-j} \Gamma
$$

Note that $\sigma(x)$ is convergent for almost all directions $\theta=x_{0} / x_{1}$ or $\theta=x_{1} / x_{0}, 0 \leq \theta \leq 1$.

Since on $\Sigma^{2}$ the relation $x_{2}=1-x_{0}-x_{1}$ holds, we restrict our attention to the first coordinates, i.e. to the domain $\left\{\left(x_{0}, x_{1}\right): 0 \leq x_{0}\right.$, $\left.0 \leq x_{1}, 0 \leq x_{0}+x_{1} \leq 1\right\}$.

Theorem. The function

$$
h\left(x_{0}, x_{1}\right)=\frac{1}{x_{0}\left(x_{0}+x_{1}\right)\left(1-x_{0}-x_{1}-\sigma\left(x_{0}, x_{1}\right)\right)}
$$

is an invariant density for $T$ restricted to $\Gamma$.

Proof. The map $T$ restricted to $\Gamma$ has only two inverse branches

$$
\begin{gathered}
V(\varepsilon)\left(x_{0}, x_{1}\right)=\left(\frac{x_{0}}{1+2 x_{0}+x_{1}}, \frac{x_{0}+x_{1}}{1+2 x_{0}+x_{1}}\right), \\
V(01)\left(x_{0}, x_{1}\right)=\left(\frac{x_{0}+x_{1}}{1+2 x_{0}+x_{1}}, \frac{x_{0}}{1+2 x_{0}+x_{1}}\right) .
\end{gathered}
$$

Then

$$
\begin{aligned}
h\left(V_{0}(\varepsilon)\left(x_{0}, x_{1}\right)\right) \omega\left(\varepsilon, x_{0}, x_{1}\right)+h\left(V(01)\left(x_{0}, x_{1}\right)\right) \omega\left(01 ; x_{0}, x_{1}\right) \\
=\frac{1}{x_{0}\left(2 x_{0}+x_{1}\right)\left(1-\left(1+2 x_{0}+x_{1}\right) \sigma\left(\frac{x_{0}}{1+2 x_{0}+x_{1}}, \frac{x_{0}+x_{1}}{1+2 x_{0}+x_{1}}\right)\right)}
\end{aligned}
$$




$$
+\frac{1}{x_{0}\left(2 x_{0}+x_{1}\right)\left(1-\left(1+2 x_{0}+x_{1}\right) \sigma\left(\frac{x_{0}}{1+2 x_{0}+x_{1}}, \frac{x_{0}+x_{1}}{1+2 x_{0}+x_{1}}\right)\right)} .
$$

We note the following properties of the function $\sigma$ :

$$
\begin{aligned}
\sigma\left(\lambda y_{0}, \lambda y_{1}\right) & =\lambda \sigma\left(y_{0}, y_{1}\right), \\
\sigma\left(y_{0}, y_{1}\right) & =\sigma\left(y_{1}, y_{0}\right), \\
\sigma\left(x_{0}, x_{0}+x_{1}\right) & =x_{0}+x_{1}+\sigma\left(x_{0}, x_{1}\right) .
\end{aligned}
$$

Therefore

$$
\left(1+2 x_{0}+x_{1}\right) \sigma\left(\frac{x_{0}}{1+2 x_{0}+x_{1}}, \frac{x_{0}+x_{1}}{1+2 x_{0}+x_{1}}\right)=x_{0}+x_{1}+\sigma\left(x_{0}, x_{1}\right) .
$$

Hence

$$
h\left(V(\varepsilon)\left(x_{0}, x_{1}\right)\right) \omega\left(\varepsilon ; x_{0}, x_{1}\right)+h\left(V(01)\left(X_{0}, x_{1}\right)\right) \omega\left(01 ; x_{0}, x_{1}\right)=h\left(x_{0}, x_{1}\right) .
$$

Remark 1. The set $\Gamma$ can be described as consisting of all needles emanating from $(0,0)$ which are given by the equations

$$
x_{0}=\lambda, \quad x_{1}=\lambda \theta, \quad 0 \leq \lambda \leq \frac{1}{1+\theta+S(\theta)}
$$

or

$$
\begin{gathered}
x_{0}=\lambda \theta, \quad x_{1}=\lambda, \quad 0 \leq \lambda \leq \frac{1}{1+\theta+S(\theta)}, \\
S(\theta)=\sigma(\theta, 1)=\sigma(1, \theta), \quad 0 \leq \theta \leq 1 .
\end{gathered}
$$

Therefore the equation

$$
x_{0}+x_{1}+\sigma\left(x_{0}, x_{1}\right)=1
$$

can be viewed as referring to the boundary of $\Gamma$ in some sense (the other parts of the boundary are given by $x_{0}=0$ and $x_{1}=1$ ).

Remark 2. This remark concerns the paper SCHWEIGER [3]. In this paper the Selmer algorithm $S$ and the Fully Subtractive algorithm $T$ were considered. The following theorem was proved:

Theorem. Let $\Gamma=\left(x_{1}, x_{2}\right) \in B^{2}:(S \circ T)^{j} x \in E, j \geq 0$. Then $\lambda(\Gamma)>0$.

The proof given was a modification of SCHWEIGER [2]. The essential idea is to show that

$$
\frac{q_{n}}{A_{n}} \geq \gamma>0, \quad \gamma=\gamma(u) \quad \text { a.e. }
$$


However in contrast to the Parry-Daniels map it is easy to show that there is a constant $\gamma>0$ such that for all $u$

$$
\frac{q_{n}}{A_{n}} \geq \gamma>0 \text {. }
$$

From

$$
a_{n+1} \leq \frac{q_{n+1}}{q_{n}}
$$

one sees by induction that

$$
q_{n} \leq\left(2+\frac{1}{q_{1}}+\cdots+\frac{1}{q_{n-1}}\right) A_{n}
$$

holds. This implies that the set $\Gamma$ contains a triangle. Therefore the set $\Gamma$ is less "exceptional" as explained in Remark 2 . In fact, $\Gamma$ contains the triangle with the vertices $(0,0),\left(\frac{1}{2}, 0\right),\left(\frac{1}{3}, \frac{1}{3}\right)$. But it is easy to see that $\Gamma$ contains at least countably many segments which start at $(0,0)$ but go beyond the line $2 x_{1}+x_{2}=1$.

The restriction of $S \circ T$ on $\Gamma$ has the $\sigma$-finite invariant measure with density

$$
h\left(x_{1}, x_{2}\right)=\frac{1}{x_{1} x_{2}\left(1-2 x_{1}-x_{2}\right)} .
$$

\section{Acknowledgement}

This paper was inspired by discussions on the dynamics of $T$ on $\Gamma$ during the Workshop on Dynamical Systems and Number Theory in Strobl (July 2007).

\section{References}

[1] NogueIRA, A. (1995) The three-dimensional Poincaré continued fraction algorithm. Israel J. Math. 90: 373-401

[2] SchweIger, F. (1981) On the Parry-Daniels transformation. Analysis 1: 171175

[3] SchweIgER, F. (2004) Ergodic and Diophantine properties of algorithms of Selmer type. Acta Arithm. 114: 99-111

Author's address: Prof. Dr. Fritz Schweiger, Department of Mathematics, University of Salzburg, Hellbrunner Strasse 34, 5020 Salzburg, Austria. E-Mail: fritz.schweiger@sbg.ac.at. 